Psychopharmaka-Verordnungen

\title{
Ergebnisse und Kommentare zum Arzneiverordnungsreport 2004
}

Jürgen Fritze, Pulheim

psychoneuro 2005; 31 (1): 46-52

Auch in diesem Jahr gibt der jährliche Arzneiverordnungsreport (Schwabe U, Paffrath D (Hrsg). Arzneiverordnungsreport 2004. Berlin, Heidelberg: Springer-Verlag, 2004, ISBN 3-540-21359-7) wichtige und spannende Informationen über das Verordnungsverhalten der niedergelassenen Ärzteschaft. Der Report übt im Vergleich zu früheren Ausgaben eine neue, eher nüchtern-wissenschaftliche Zurückhaltung in seinen Interpretationen. Allerdings fallen doch gelegentlich Widersprüche auf, die vermutlich den unterschiedlichen Autorenschaften zuzuschreiben sind.

D ie Ausgaben der GKV für Arzneimittel sind - infolge mit dem Beitragssatzsicherungsgesetz eingeführter Rabatte - 2003 gegenüber 2002 um 1,1\% auf 21,1 Mrd. Euro (ohne Zuzahlungen der Versicherten) gesunken. Dazu tragen die Psychopharmaka (denen - abweichend vom Report - nicht nur Neuroleptika, Lithium, Antidepressiva und Anxiolytika zuzuordnen sind, sondern auch Hypnotika und Antidementiva) 6,7\% bei, womit sie Rang 3 der häufigst verordneten Arzneimittelgruppen einnehmen. Erstmals bietet der Report Informationen über das Verordnungsverhalten in einzelnen Fachgebieten, wobei Neurologie einerseits und Psychiatrie und Psychotherapie andererseits nicht differenziert werden konnten. Mit einem Umsatzanteil an den Gesamtausgaben von 6,9\% (1,67 Mrd. Euro) stehen die Nervenärzte an 3. Stelle nach Allgemeinärzten $(31 \%)$ und Internisten (37\%). 60\% der Ausgaben der Nervenärzte entfallen auf neurologische Indikationen. Das Einsparpotential der Nervenärzte, das durch verstärkte Bevorzugung von Generika, Verzicht auf die Verordnung von sog. Analogpräparaten („me-too-Präparate“) und Verzicht auf „umstrittene“ Arzneimittel zu realisieren sein soll, schätzt der Report auf 156 Mio. Euro (9,4\%) gegenüber 18,6\% im Durchschnitt aller Fachgebiete. Das gesamte Einsparpotential schätzt der Report auf 4,5 Mrd. Euro (Vorjahr 4,1 Mrd. Euro), also $18,4 \%$ der Gesamtausgaben (inklusive Zuzahlungen) für die ambulante Arzneimitteltherapie der GKVVersicherten.

Erstmals weist der Report auch Auswertungen nach Alter und Geschlecht aus, wonach Psychopharmaka (in der Konnotation des Reports Tranquillantien, Antidepressiva und Neuroleptika) unterhalb des 20. Lebensjahres nur bei ca. $2 \%$ verordnet keine detaillierteren Informationen.

Neue Neuropsychopharmaka Unter den 17 im Jahre 2003 neu zugelassenen Wirkstoffen („Fricke werden. Leider bietet der Report
Liste“) findet sich als einziges Neuropsychopharmakon Escitalopram $\left(\right.$ Cipralex $\left.^{\circledR}\right)$ mit den Indikationen Depression, Panikstörung und (seit 2004) soziale Phobie. Der Report ordnet Escitalopram den „Analogpräparaten mit keinen oder nur marginalen Unterschieden zu bereits eingeführten Präparaten“ zu. Die zugrunde gelegten Argumente sind leider dürftig, indem der Report allein auf der Basis einer einzigen, rein qualitativen Übersichtsarbeit (22) ohne sorgfältige Würdigung der übrigen Literatur erklärt, die „Werbeaussagen zu Escitalopram sind nicht gerechtfertigt", Citalopram sei zu bevorzugen. Escitalopram stellt das S-Enantiomer des razemischen Citaloprams dar. Das kann zu einer derart kurzschlüssigen Aussage verführen. Jedoch haben inzwischen drei, systematische Meta-Analysen $(1,13,18)$ gezeigt, dass Escitalopram dem Citalopram - bei äquivalenten Dosierungen - signifikant überlegen ist. Dies erklärt sich daraus, dass das REnantiomer die Wirkung des SEnantiomers behindert. Entsprechend ist von einer Dosissteigerung des Escitaloprams - kaum aber des razemischen Citaloprams - eine zunehmende Wirkung zu erwarten. Damit stellt Escitalopram eine klinisch relevante Verbesserung dar. 


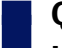

Qualität der Versorgung

und Einsparpotentiale

Die Qualität der Versorgung ist im Verordnungsreport 2004 - wie 2003 und im Gegensatz zu Vorjahren kein explizites Thema. Immer noch wenig pharmakologisch und sehr ökonomisch motiviert sind die Vorschläge zur Substitution im Bereich der sog. Analogpräparate bei den Antidepressiva. Unverändert können die Substitutionsvorschläge auch lebensbedrohliche Folgen zeitigen: $\mathrm{Ci}$ talopram und Sertralin sollen durch Fluoxetin substituiert werden. Dabei wird in einem anderen Kapitel ausdrücklich u.a. auf das hohe Interaktionspotential von Fluoxetin hingewiesen, weshalb „Citalopram und Sertralin diesbezüglich günstiger" seien und ihre Bevorzugung „grundsätzlich als sinnvoll zu bewerten".

Die Verordnung von Nootropika wie Ginkgo biloba, Dihydroergotoxin, Piracetam geiselt der Report als „Zeichen einer beträchtlichen Fehlversorgung" und schlägt vor, die hierfür im Jahr 2003 aufgewendeten Mittel (117 Mio. Euro) für die Behandlung von Alzheimerkranken mit Cholinesterasehemmern und Memantin zu verwenden.

\section{Generika}

Inzwischen haben Generika im Gesamtmarkt einen Verordnungsanteil von 54\% und einen Umsatzanteil von $30 \%$. Im generikafähigen Markt ist der Verordnungsanteil der Generika mit 75,3\% stabil geblieben, während der Umsatzanteil mit 67,5\% um absolut $1 \%$ gefallen ist. Wie hoch der Generika-Anteil im generikafähigen Antidepressiva- und Neuroleptika-Markt ist, lässt sich dem Arzneiverordnungsreport leider nicht direkt entnehmen, da nur über die umsatzstärksten Fertigarzneimittel berichtet wird. Immerhin liegen soweit aus den Daten ableitbar - die Anteile hoch (Tab. 1) und steigen über die Jahre.

\section{Verordnungsspektren}

Antidepressiva

Die Anzahl verordneter Tagesdosen (DDD) von Antidepressiva (DDD +7\%; chemisch definierte $12 \%$ ) ist bei allgemeiner Abnahme (-2\%) der Verordnungen weiter gestiegen (Abb. 1),

\section{Tab. 1 Anteile generischer Fertigarzneimittel einiger anti- depressiver und neuroleptischer Wirkstoffe an den Gesamtverordnungen und Umsätzen}

\begin{tabular}{|c|c|c|c|c|c|c|}
\hline \multirow[b]{2}{*}{ Wirkstoffe } & \multicolumn{3}{|c|}{ Verordnungen (DDD) } & \multicolumn{3}{|c|}{ Umsätze (Euro) } \\
\hline & 2001 & 2002 & 2003 & 2001 & 2002 & 2003 \\
\hline \multicolumn{7}{|l|}{ - Antidepressiva } \\
\hline Fluoxetin & & $77 \%$ & $90 \%$ & & $66 \%$ & $76 \%$ \\
\hline Doxepin & $74 \%$ & $74 \%$ & $79 \%$ & $65 \%$ & $66 \%$ & $74 \%$ \\
\hline Paroxetin & & $57 \%$ & $77 \%$ & & $52 \%$ & $74 \%$ \\
\hline Amitriptylin & $62 \%$ & $66 \%$ & $76 \%$ & $56 \%$ & $62 \%$ & $75 \%$ \\
\hline Citalopram & & & $68 \%$ & & & $58 \%$ \\
\hline Maprotilin & & $51 \%$ & $62 \%$ & & $48 \%$ & $61 \%$ \\
\hline Amitriptylinoxid & & $50 \%$ & $54 \%$ & & $46 \%$ & $50 \%$ \\
\hline Trimipramin & & $46 \%$ & $49 \%$ & & $40 \%$ & $46 \%$ \\
\hline Clomipramin & & $15 \%$ & $20 \%$ & & $13 \%$ & $18 \%$ \\
\hline \multicolumn{7}{|l|}{ - Neuroleptika } \\
\hline Sulpirid & & $85 \%$ & $89 \%$ & & $82 \%$ & $85 \%$ \\
\hline Melperon & & $78 \%$ & $83 \%$ & & $73 \%$ & $81 \%$ \\
\hline Promethazin & & & $68 \%$ & & & $62 \%$ \\
\hline Clozapin & & $61 \%$ & $65 \%$ & & $54 \%$ & $58 \%$ \\
\hline Perazin & $57 \%$ & $60 \%$ & $64 \%$ & & $56 \%$ & $59 \%$ \\
\hline Levomepromazin & $54 \%$ & $57 \%$ & $59 \%$ & & $54 \%$ & $55 \%$ \\
\hline Benperidol & & & $59 \%$ & & & $55 \%$ \\
\hline Chlorprothixen & & & $45 \%$ & & & $41 \%$ \\
\hline Haloperidol & $43 \%$ & $41 \%$ & $41 \%$ & & $31 \%$ & $31 \%$ \\
\hline Thioridazin & & & $22 \%$ & & & $29 \%$ \\
\hline Pipamperon & & & $22 \%$ & & & $21 \%$ \\
\hline
\end{tabular}

was auf einen weiteren Rückgang der Unterbehandlung depressiv Kranker hinweist. Die verordneten Tagesdosen (DDD) von Antidepressiva haben stärker zugenommen als die Tagesdosen generell (3,3\%). Die Umsatz- steigerung (+4\%) ist erstmals seit Jahren unterproportional im Vergleich zur generellen Umsatzsteigerung (+6\%; Abb. 1), obwohl die Verordnung moderner Antidepressiva weiter zugenommen hat (Abb. 2).

\section{Abb. 1 Arzneiverordnungen und -umsätze zu Lasten der GKV}

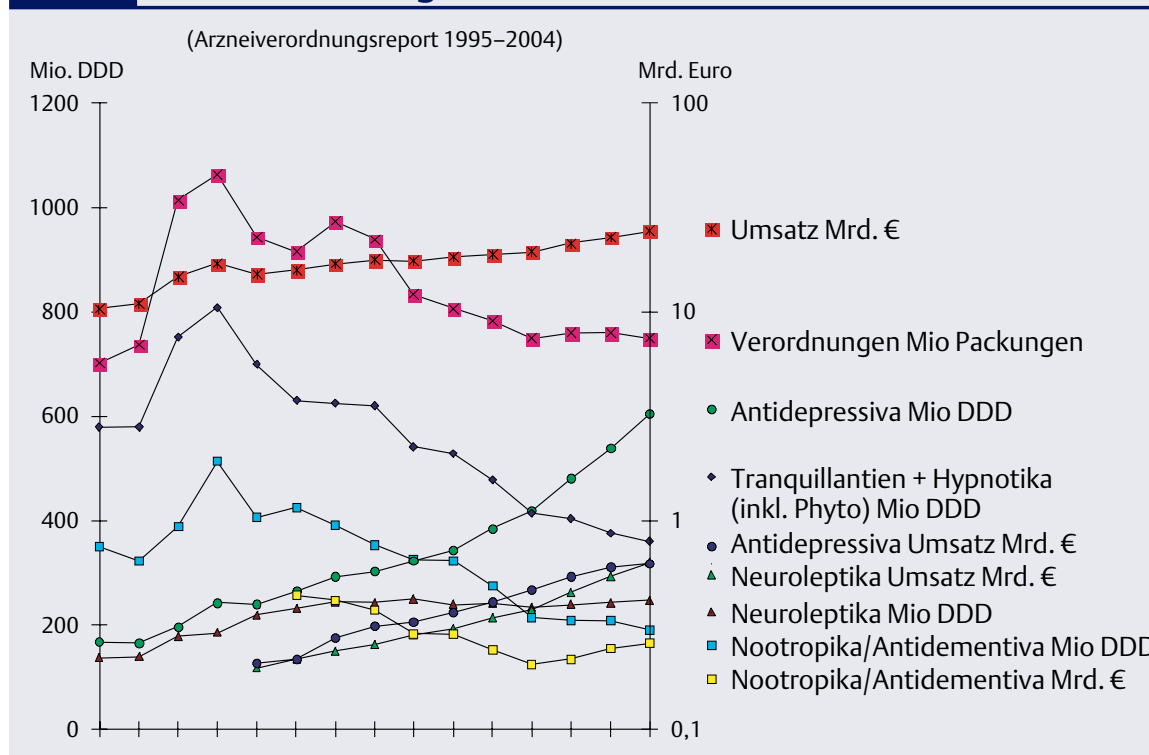

$\begin{array}{lllllllllllllll}1989 & 90 & 91 & 92 & 93 & 94 & 95 & 96 & 97 & 98 & 99 & 00 & 01 & 02 & 2003\end{array}$

Jahr 


\begin{tabular}{|c|c|c|c|c|c|c|c|}
\hline Rang & & 1980 & 1985 & 1990 & 1995 & $1998 / 2002$ & $\begin{array}{l}\text { Differenz } \\
\text { (Maximum 1998/2002) }\end{array}$ \\
\hline 1 & Dänemark & 31,6 & 27,9 & 23,9 & 17,7 & 14,4 & $-54 \%$ \\
\hline 2 & Ungarn & 44,9 & 44,4 & 39,9 & 32,9 & 28 & $-38 \%$ \\
\hline 3 & Deutschland & 20,8 & 16,5 & 17,8 & 15,8 & 13,6 & $-35 \%$ \\
\hline 4 & Österreich & 25,7 & 27,7 & 23,6 & 22,2 & 18,3 & $-34 \%$ \\
\hline 5 & Estland & 36,7 & 22,3 & 27,1 & 40,1 & 27,5 & $-31 \%$ \\
\hline 6 & Schweiz & 25,7 & 25 & 21,9 & 20,2 & 18,1 & $-30 \%$ \\
\hline 7 & Schweden & 19,4 & 18,2 & 17,2 & 15,3 & 13,8 & $-29 \%$ \\
\hline 8 & Finnland & 25,7 & 24,6 & 30,3 & 27,2 & 22,5 & $-26 \%$ \\
\hline 9 & Tschechien & - & 20,9 & 19,3 & 17,5 & 16,1 & $-23 \%$ \\
\hline 10 & Frankreich & 19,4 & 22,5 & 20 & 20,6 & 17,5 & $-22 \%$ \\
\hline
\end{tabular}

Die modernen Antidepressiva haben inzwischen einen Anteil von ca. $42 \%$ (2002: 37\%) der gesamten (einschließlich niedrig dosierter Neuroleptika und JohanniskrautExtrakte) Antidepressiva-Verordnungen (DDD; Abb. 2) und ca. 64\% (2002: 60\%) am Umsatz (Abb. 3). Der Anteil der verordneten Tagesdosen (DDD) moderner Antidepressiva an den chemisch definierten Antidepressiva im engeren Sinne liegt bei 50\% (2002: 46\%), der Anteil ihres Umsatzes bei $71 \%$ (2002: 68\%). Wie anderenorts (7) beschrieben, wäre nach medizinischen Kriterien zu erwarten, dass der Verordnungsanteil der modernen Antidepressiva an den Antide-
pressiva-Gesamtverordnungen bei annähernd $50 \%$ läge.

Der Arzneiverordnungsreport widmet sich auch dem seit Sommer 2003 erneut aktuellen Problem der Häufigkeit suizidaler Ereignisse (das sind suizidale Gedanken, selbstschädigende Handlungen und Suizide) unter selektiv-serotonergen Antidepressiva (SSRI) im Vergleich zu anderen Antidepressiva. Der Report tut dies leider in etwas unausgewogener Weise, indem er allein die Studie von Donovan et al. (6) und die Meta-Analyse von Healy (16) berücksichtigt und daraus ableitet: „Unter SSRI wurden deutlich mehr suizidale Handlungen als unter NSMRI (nicht-selektive Monoaminrückaufnahmehem-

\section{Abb. 2 Verteilung der Antidepressiva-Verordnungen (DDD) zu Lasten der GKV}

(Arzneiverordnungsreport 1995-2004)

( ${ }^{*}$ inklusive niedrig dosierte Neuroleptika)

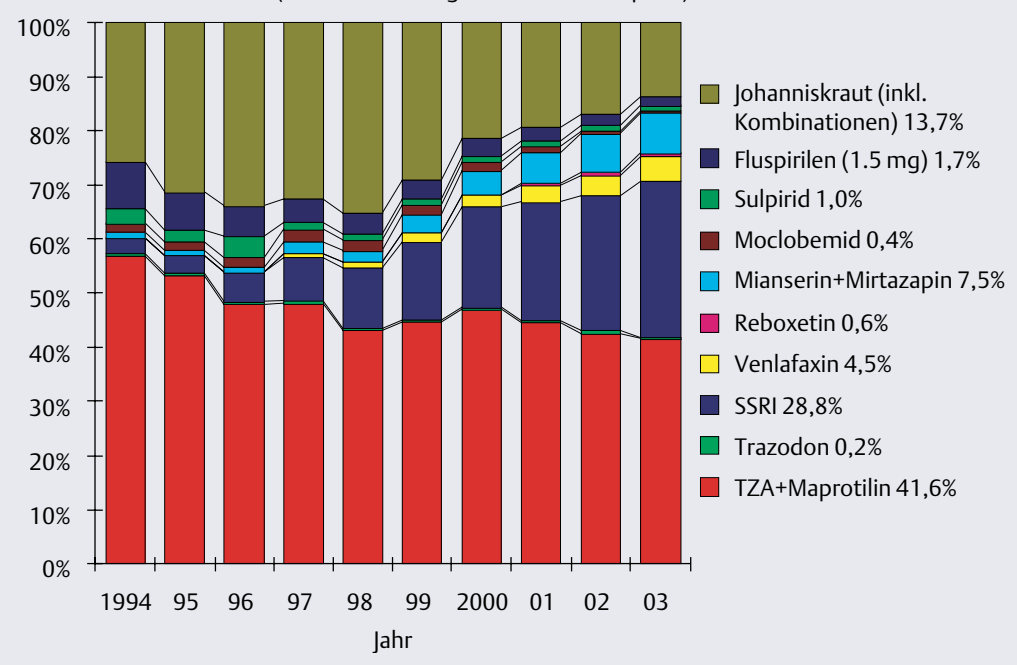

mer) beobachtet. Donovan et al. (6) beobachteten unter Alltagsbedingungen (prospektiv erhobene Daten einer Notfalleinrichtung) unter selektiv serotonergen Antidepressiva signifikant $(\mathrm{p}<0,001)$ mehr selbstschädigende Handlungen je 10000 Verordnungen eines Antidepressivums als unter trizyklischen Antidepressiva. Die Inzidenz selbstschädigender Handlungen war am höchsten unter Fluoxetin, am geringsten unter Amitriptylin. So eindrucksvoll die Studie von Donovan et al. (6) ist, so können die Ergebnisse aber ein Artefakt darstellen, z.B. indem suizidgefährdeten Patienten aus Sorge vor der Toxizität trizyklischer Antidepressiva in besonderem Maße SSRI verordnet wurden. Jedenfalls beweisen sie kein erhöhtes Suizidrisiko unter SSRI.

Healy (16) fand bei einer Reanalyse randomisierter Studien - inklusive Daten der FDA aus Zulassungsstudien - unter den selektiv-serotonergen Antidepressiva eine Rate suizidaler Handlungen von 1,55\% (Versuche $1,39 \%$, Suizide $0,15 \%$ ), unter den Vergleichssubstanzen (im wesentlichen trizyklische Antidepressiva, TZA) $0,79 \%$ (Versuche 0,65\%, Suizide 0,14\%), unter Plazebo $0,67 \%$ (Versuche 0,65\%, Suizide $0,04 \%$ ). Für suizidale Handlungen betrug der Odds-Ratio OR=2,0 (95\% CI 1,2-3,3) im Vergleich zwischen den selektiv-serotonergen Antidepressiva und Plazebo sowie $\mathrm{OR}=3,1$ (95\% CI 0,4-23,1) für Suizide. Jedenfalls unterscheiden sich SSRI und Referenzantidepressiva) nicht in den Raten vollendeten Suizides.

Im Gegenteil weisen inzwischen einige pharmakoepidemiologische Studien auf einen Zusammenhang zwischen zunehmender Verordnung von Antidepressiva und abnehmender Suizidrate hin. Eine australische Studie (15) fand von 1991 bis 2000 bei weitgehend konstanter genereller Suizidrate - eine Assoziation zwischen der Verordnung von Antidepressiva und abnehmender Suizidrate nach Adjustierung für Alterskohorten. Olfson et al. (20) fanden bei Jugendlichen in den USA in der Periode 1990 bis 2000 nach Adjustierung für konfundierende Variablen wie Geschlecht, Alter, soziale Schicht, ethnischer Ursprung einen 
inversen Zusammenhang zwischen regionalen Änderungen der Suizidraten und Änderungen der Verordnung von Antidepressiva. Gunnell et al. (14) fanden in Großbritannien in der Periode 1950 bis 1998, dass rückläufige Suizidraten im höheren Lebensalter mit zunehmender Verordnung von Antidepressiva, steigendem Bruttoinlandsprodukt, Heirat und Berufstätigkeit der Frauen assoziiert war. Carlsten et al. (3) fanden in Schweden in der Periode 1977 bis 1997 einen mit der Einführung der SSRI (1990) beschleunigte und von der Verordnungsrate abhängige Abnahme der Suizidrate in den meisten Altersgruppen. Auch in Ungarn nahm die Suizidrate mit steigendem Einsatz von Antidepressiva $\mathrm{ab}$ (21), und dies trotz zunehmender Arbeitslosigkeit und trotz steigenden Alkoholkonsums, beides Risikofaktoren für Suizid. Keinen solchen Zusammenhang konnten allerdings Barbui et al. (2) in Italien (19861997) für Männer finden. Und es ist banal darauf hinzuweisen, dass eine Assoziation nicht mit einem Kausalzusammenhang gleichzusetzen ist. Tabelle 2 gibt einen Überblick über die Entwicklung der Suizidraten im internationalen Vergleich.

Bei Kindern und Jugendlichen bei denen sich die neuerliche Diskussion an im Vergleich zu Plazebo erhöhten Raten suizidaler Ereignisse, nicht aber vollendeter Suizide, entzündete - ist die Frage suizidaler Handlungen insofern besonders bedeutsam, als der eindeutige Wirksamkeitsnachweis in der Indikation Depression bisher nicht erbracht werden konnte (17). Das bedeutet nicht, die Unwirksamkeit wäre belegt. Das bisherige Scheitern kann an methodischen Problemen, insbesondere der Heterogenität der Kollektive, an hier ausgesprochen hohen Plazebo-Responseraten und begrenzter Änderungssensitivität der Messinstrumente, liegen. Jedenfalls ist bei Kindern und Jugendlichen die Indikation besonders sorgfältig abzuwägen (und zu dokumentieren). Es gilt immer, von Beginn an eine suizidpräventive therapeutische Beziehung aufzubauen und, insbesondere bei fortbestehender Depressivität, Suizidalität engmaschig zu

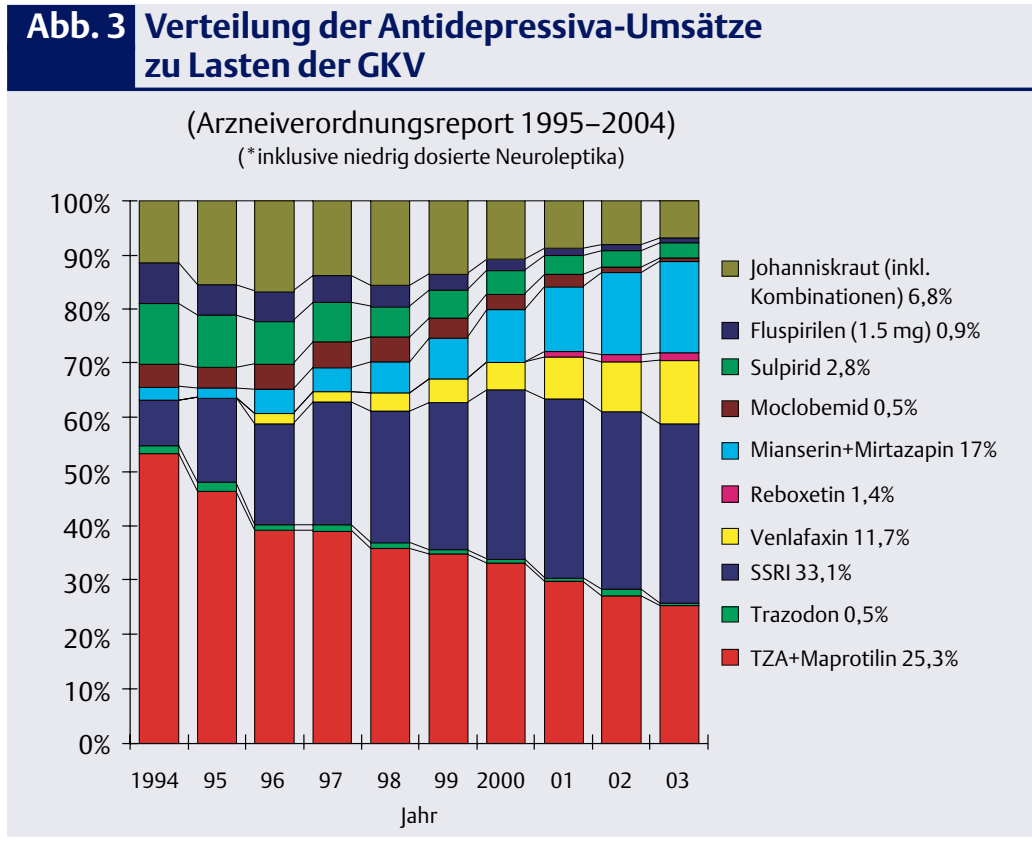

prüfen. Dabei ist auf Akathisie-ähnliche Beschwerden als potentielle Nebenwirkung besonders zu achten und ggf. zu intervenieren.

\section{Neuroleptika}

Die Verordnung von Neuroleptika ist weitgehend stabil geblieben. Die Definition von DDD ist für Neuroleptika besonders schwierig und variiert von Jahr zu Jahr, so dass die nominale Zunahme von $+2 \%$ nicht sicher interpretiert werden kann. Die erneute Umsatzsteigerung $(+16 \%$,
2002: $+19 \%$ ) ist überproportional im Vergleich zur generellen Umsatzsteigerung (6\%; Abb. 1), was einem vermehrten Einsatz der modernen, nebenwirkungsärmeren (und teureren) atypischen Neuroleptika entspricht. Das darf auch dahingehend interpretiert werden, dass schizophren Kranken grundsätzlich die Teilhabe am Fortschritt nicht selektiv vorenthalten wird.

Die atypischen Neuroleptika (Abb. 4) im engeren Sinne haben mit einem Anteil von 31\% gegenüber

\section{Abb. 4 Verteilung der Neuroleptika-Verordnungen (DDD*) zu Lasten der GKV}

(Arzneiverordnungsreport 1995-2004)

( ${ }^{*}$ Veränderungen über die Zeit z.T. bedingt durch Änderungen der Definition der DDD)

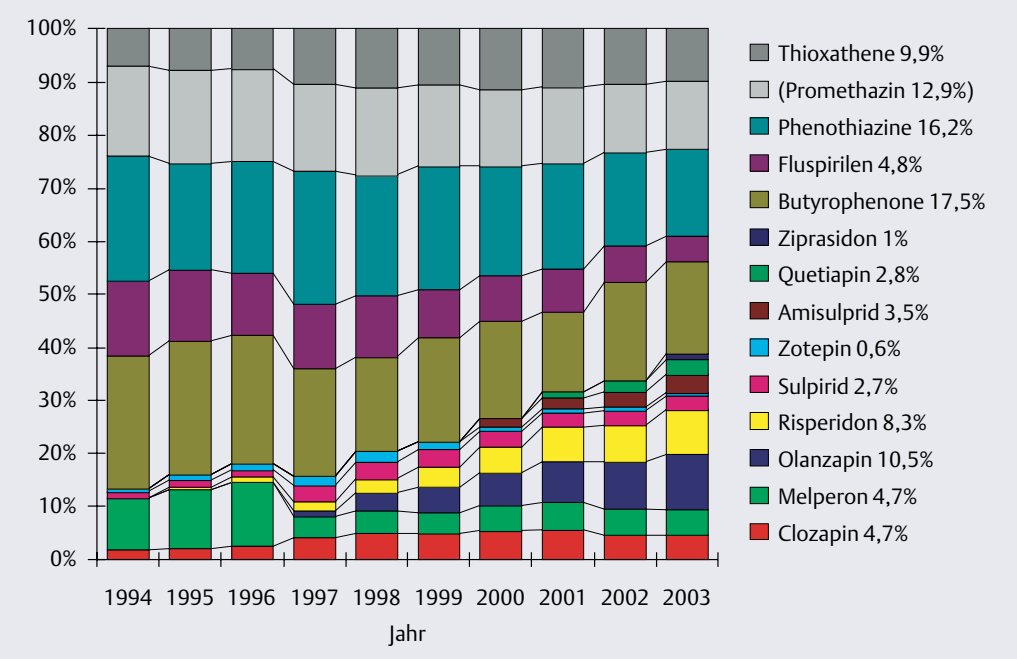




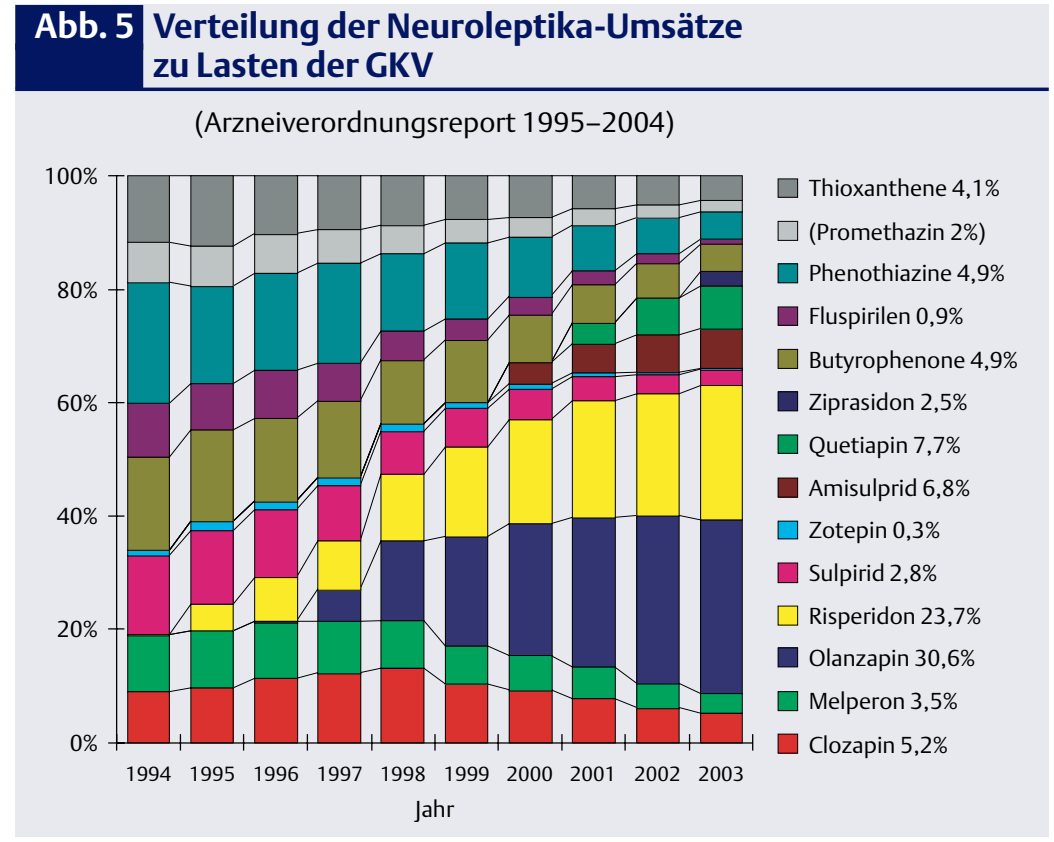

2002 bei den verordneten Tagesdosen um 5\%-Punkte gewonnen (ca. 77\% des Umsatzes (2002: 70\%, Abb. 5). Die atypischen Neuroleptika setzen sich also trotz Kostendrucks und der damit verbundenen persönlichen Risiken für den Arzt weiter durch. Wie anderenorts (8) beschrieben, wäre nach medizinischen Kriterien zu erwarten, dass der Anteil der verordneten Tagesdosen der atypischen Antipsychotika an den Neuroleptika-Gesamtverordnungen bei annähernd 25\% läge. Damit überschreitet der Anteil seit dem Jahr 2002 den Schätzwert. Diese Schätzung ging davon aus, dass Patienten, die mit konventionellen Neuroleptika gut eingestellt sind, nicht auf atypische Neuroleptika umgestellt werden.

Zutreffend stellt der Report fest, dass die therapeutische Überlegenheit und Kosteneffektivität atypischer Neuroleptika nicht abschließend belegt ist. Atypische und typische Neuroleptika bilden ein Kontinuum von mehr zu geringerer „Aty-

\section{Abb. 6 Verteilung der Verordnungen (DDD) von Nootropika/- Antidementiva zu Lasten der GKV}

(Arzneiverordnungsreport 1995-2004)

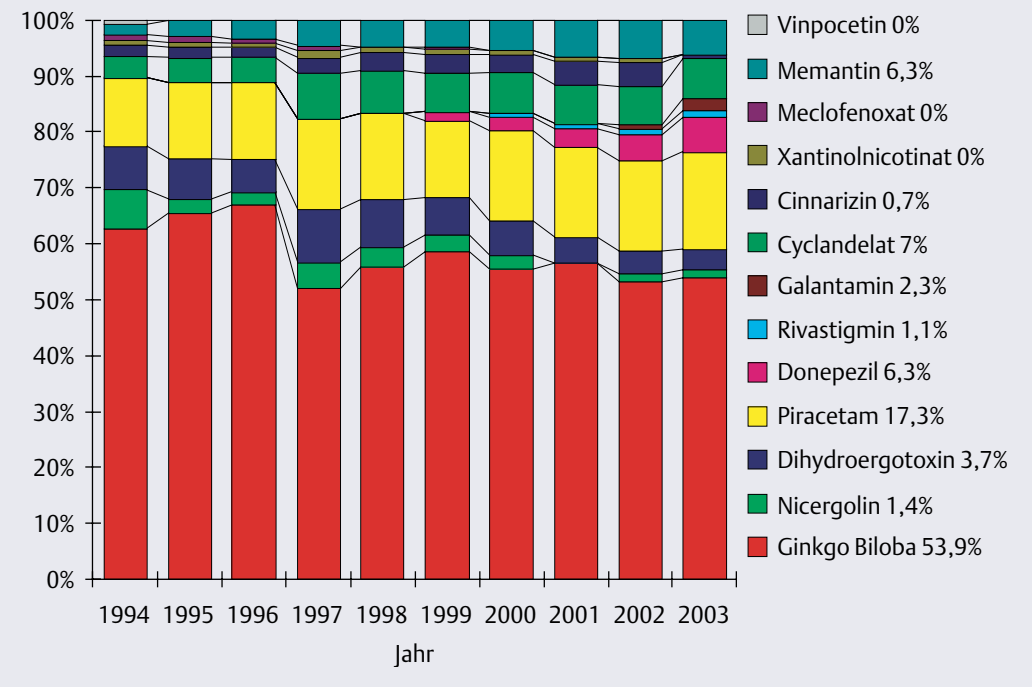

pikalität“. Entscheidend für die Indikation sind die Verträglichkeitsvorteile. Tatsächlich wurde in zahlreichen Vergleichsstudien die Dosis des konventionellen Neuroleptikums (in der Regel Haloperidol) zu hoch gewählt. Das ist aber nicht als Beweis zu werten, bei nur hinreichend geringer Haloperidol-Dosis wäre die extrapyramidalmotorische Verträglichkeit identisch. Die erneut vom Report angeführte Meta-Analyse von Geddes et al. (12) hat als Verträglichkeitskriterium allein die Abbruchraten berücksichtigt und hier nicht einmal getrennt nach Abbrüchen wegen Unverträglichkeit bzw. mangelnder Wirksamkeit. Therapieabbruch ist der letzte Ausweg bei Nebenwirkungen, also kein geeigneter Parameter für Verträglichkeit. Gemäß der Meta-Analyse von Davis et al. (5) waren unabhängig von der Dosis Clozapin, Amisulprid, Risperidon und Olanzapin signifikant wirksamer als die konventionellen Neuroleptika. Die Verträglichkeit war ebenfalls besser. Am Ende ist bei der Auswahl entscheidend, welche Präferenzen nach Aufklärung über Risiken und Nebenwirkungen der Patient äußert.

\section{Antidementiva/Nootropika}

Trotz seit Jahren und weiter rückläufiger Verordnungen (-8\%; Abb. 1) sind die Umsätze (Abb. 1) der Antidementiva $(+6 \%)$ gestiegen (erstmals 2001). Die Cholinesterasehemmer haben bei der Alzheimer-Demenz die Kranken dennoch unverändert nicht sachgerecht erreicht: Donepezil, Rivastigmin und Galantamin (im Jahr 2001 zugelassen) hatten im Jahre 2003 einen Anteil von nur 9,7\% (2002: $6,5 \%$ ) an den Verordnungen (DDD; Abb. 6), aber immerhin von $31 \%$ (2002: 28,6\%) am Umsatz (Abb. 7). Memantin, dem im Jahr 2002 formal die europäische Zulassung für mittelschwere bis schwere Demenz vom Alzheimer-Typ erteilt wurde, verzeichnet einen Anteil von 6,3\% an den Tagesdosen und 21,7\% an den Umsätzen. Ginkgo-biloba-Extrakte dominieren unverändert mit $54 \%$ der Tagesdosen und 30\% der Umsätze dieses Indikationsgebietes (Abb. 6 \& 7). Auch nach Würdigung der AD2000-Studie (4), die anderenorts kritisch kom- 
mentiert wurde (10), anerkennt der Report, „Acetylcholinesterasehemmer können zur Kognitionsverbesserung herangezogen werden“. Diese Option können bisher nominal ca. 15\% der Betroffenen nutzen. Folgt man der Kritik des Arzneiverordnungsreports, Ginkgo-biloba-Extrakte stellten Fehlbehandlung dar und seien zu substituieren, so bedarf es erheblicher zusätzlicher Ressourcen (9).

Unverständlich ist die Interpretation der Studie von Tariot et al. (23), in der Memantin im Vergleich zu Plazebo additiv zu einer Basistherapie mit Donepezil untersucht wurde, wobei Memantin Plazebo signifikant überlegen war. Dieser Befund ist erstaunlich und muss die Kranken hoffnungsvoll stimmen, denn eigentlich wäre im Sinne eines Deckeneffektes denkbar gewesen, dass die Besserungspotentiale bereits durch Donepezil ausgeschöpft waren. Stattdessen zweifelt der Report den additiven Effekt an. Nachvollziehbar aber wäre allein die methodische Kritik, dass in dieser Studie ein dritter Arm mit alleiniger Therapie mit Memantin fehlte.

\section{Entwöhnungsmittel}

Bereits im letzten Kommentar zum Arzneiverordnungsreport 2003 wurde auf die Widersprüche zwischen dem Ausschluss von Mitteln zur Raucherentwöhnung (Buproprion (Zyban $\left.{ }^{\circledR}\right)$; Nikotin) aus dem Leistungskatalog der GKV durch die Arzneimittelrichtlinien (AMR) des Bundesausschusses der Ärzte und Krankenkassen nach § 92 SGB V und seit 01.01.2004 auch durch das GKVModernisierungsgesetz ( $§ 34$ SGB V) hingewiesen: Der Ausschluss ist angesichts der Bedeutung des Rauchens als Risikofaktor für die führenden Todesursachen (u.a. Herzinfarkt und Malignome) medizinisch unplausibel. Er ist aber auch angesichts des Bekenntnisses der Bundesregierung zur Prävention (der Referentenentwurf des Präventionsgesetzes wurde jüngst bekannt), hier ein rauchfreies (Raucher-freies) Land zu realisieren, wenig plausibel: Der Ausschluss ist unlogisch.

Acamprosat wird in Anlage 4 der AMR ausdrücklich als verordnungsfähig genannt, wobei „zur Vermeidung eines nicht sachgerechten Ein-

\section{Abb. 7 Verteilung der Umsätze von Nootropika/Antidementiva zu Lasten der GKV}

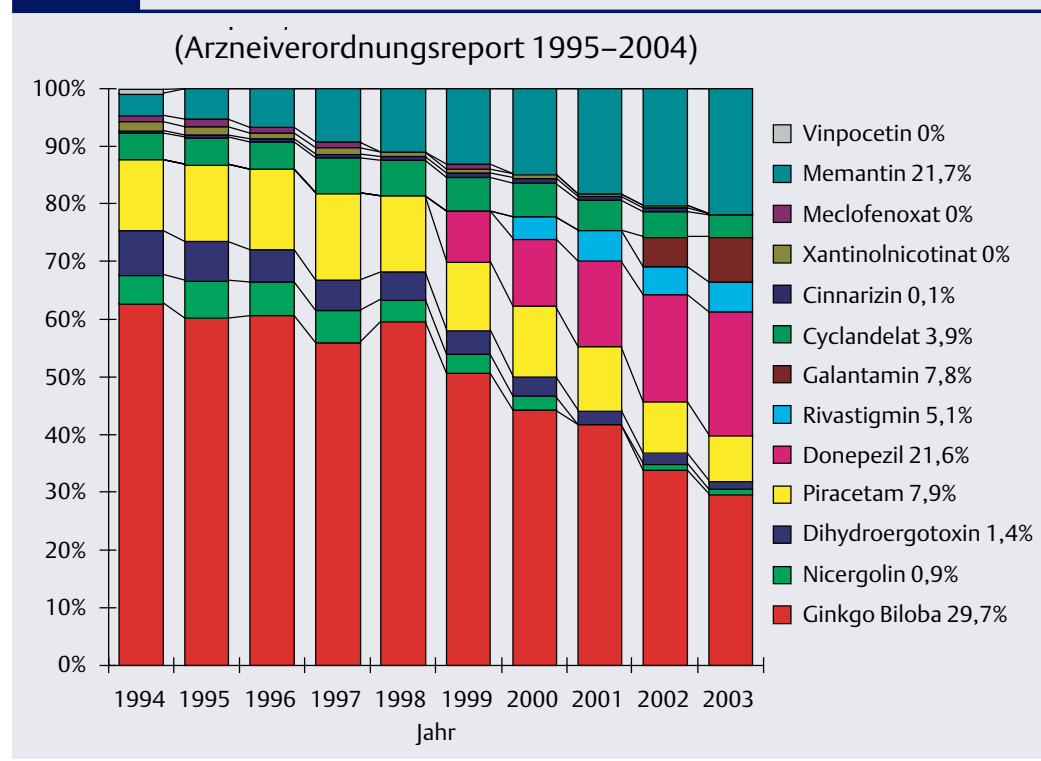

satzes auf die bestimmungsgemäße Anwendung von Acamprosat ausschließlich als Zusatztherapeutikum im Rahmen einer psychosozial betreuten Abstinenzbehandlung“ hingewiesen wird. Die Verordnungen von Acamprosat sind im Jahr 2003 erstmals wieder gestiegen. Dennoch werden allenfalls $5 \%$ der geeigneten Patienten erreicht.

Dieser Unterversorgung leistet der Arzneiverordnungsreport erneut Vorschub, indem die ausgezeichnete Datenlage weitgehend verschwiegen wird: Tatsächlich existieren inzwischen 20 doppelblinde, randomisierte Studien (19), von denen 18 eine signifikante Überlegenheit gegen Plazebo zeigten, mit einer relativen Risikoreduktion von rund $50 \%$ : nach sechs Monaten sind signifikant $(\mathrm{p}<0,001)$ mehr Kranke unter Acamprosat abstinent als unter Plazebo (Acamprosat: 36,1\%; Plazebo: 23,4\%).

\section{Psychostimulantien}

In den letzten zehn Jahren hat sich die Verordnung von Methyl-

\section{Abb. 8 Verordnung von Acamprosat}

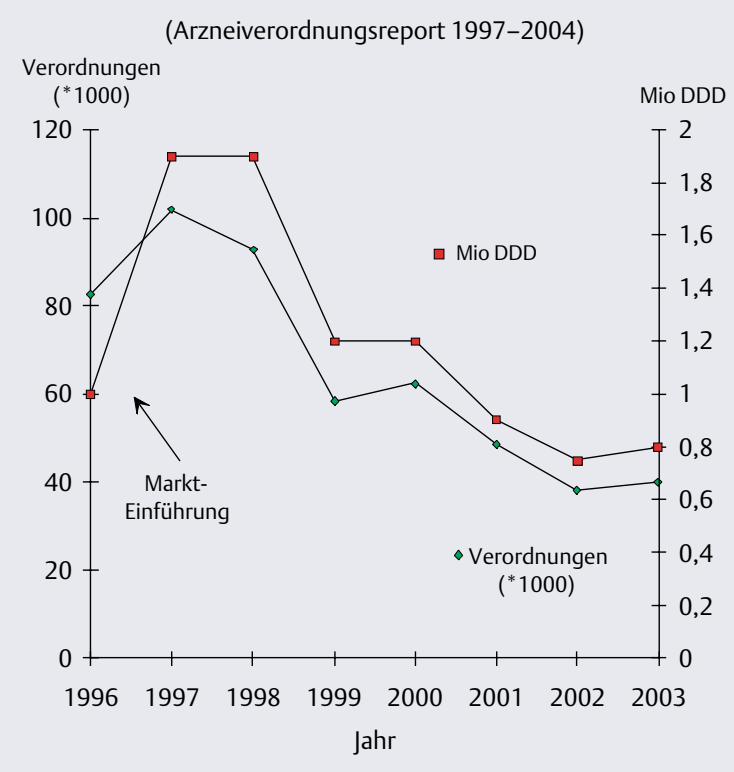




\section{Abb. 9 Verordnungen (Mio DDD) von Methylphenidat zu Lasten der GKV}

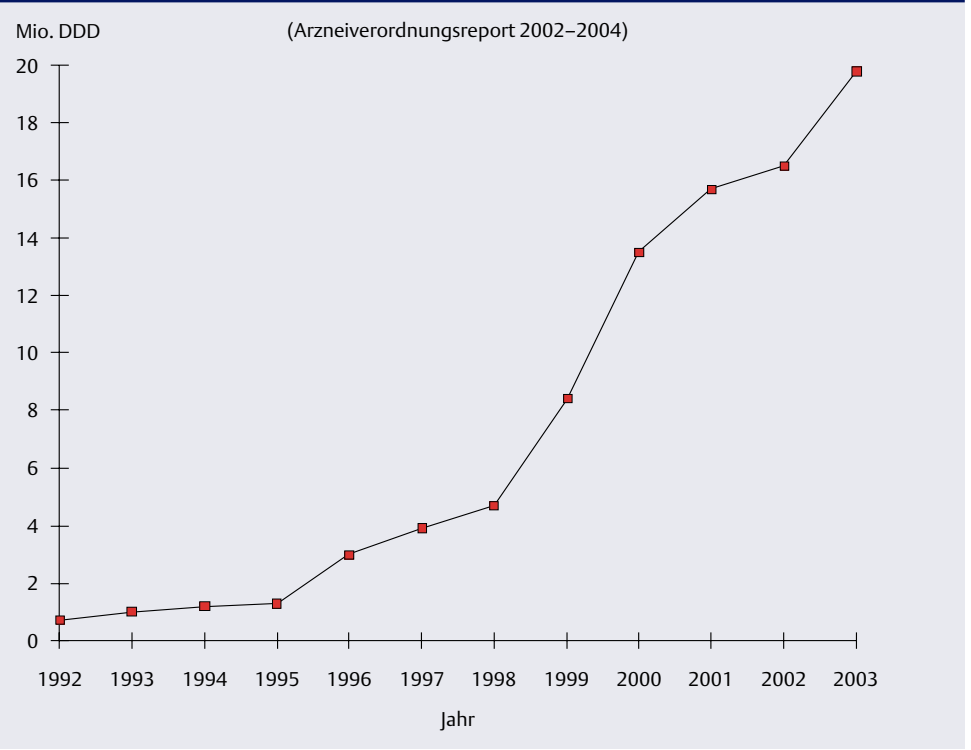

phenidat verdreißigfacht (Abb. 9). Der Report schreibt diese Zunahme dem Abbau der Unterversorgung von Patienten mit AufmerksamkeitsHyperaktivitätsstörung (ADHS) zu.

Unverändert ungelöst ist seit dem Urteil des Bundessozialgerichts (BSG) vom 19.03.2002 das Problem des angeblichen Off-Label-Use bei Verordnung von Methylphenidat an Erwachsene mit ADHS. Anderenorts (11) ist detailliert dargelegt worden, dass die Verordnung von Methylphenidat an Erwachsene keinen OffLabel-Use darstellt, wenn man die Logik des BSG zugrunde legt.

Mit dem GKV-Modernisierungsgesetz ist das zuständige Expertengremium beim Bundesinstitut für Arzneimittel und Medizinprodukte (BfArM) gesetzlich verankert worden. Leider ist noch immer nicht öffentlich erkennbar, wann sich die Expertenkommission neben der Onkologie - endlich - auch mit den Psychopharmaka befassen wird, also auch mit Methylphenidat. Diese inzwischen jahrelange Posteriorisierung der Anliegen psychisch Kranker ist rechtsstaatlich nicht vertretbar.

\section{Literatur}

1. Auquier P, Robitail S, Llorca PM, Rive B. Comparison of escitalopram and citalopram efficacy: A meta-analysis. International Journal of Psychiatry in Clinical Practice 2003; 7 : 259-268
2. Barbui C, Campomori A, D’Avanzo B, Negri E, Garattini S. Antidepressant drug use in Italy since the introduction of SSRIs: national trends, regional differences and impact on suicide rates. Soc Psychiatry Psychiatr Epidemiol 1999; 34: 152-156

3. Carlsten A, Waern M, Ekedahl A, Ranstam J. Antidepressant medication and suicide in Sweden. Pharmacoepidemiol Drug Saf 2001; 10: 525-530

4. Courtney C, Farrell D, Gray R, Hills R, Lynch L, Sellwood E, Edwards S, Hardyman W, Raftery J, Crome P, Lendon C, Shaw H, Bentham P; AD2000 Collaborative Group. Long-term donepezil treatment in 565 patients with Alzheimer's disease (AD2000): randomised double-blind trial. Lancet 2004; 363: 2105-2115

5. Davis JM, Chen N, Glick ID. A meta-analysis of the efficacy of second-generation antipsychotics. Arch Gen Psychiatry 2003; 60: 553-64

6. Donovan S, Clayton A, Beeharry M, JoJ, Madeley R. Deliberate self-harm and antidepressant drugs: Investigation of a possible link. Br J Psychiatry 2000; 177: 551-556

7. Fritze J. Innovative Therapien erfordern zusätzliche finanzielle Ressourcen - Teil III (Antidepressiva). Neurotransmitter 2001; 12(3): 33-39

8. Fritze J. Innovative Therapien erfordern zusätzliche finanzielle Ressourcen - Teil I (Antipsychotika). Neurotransmitter 2001; 12(1): 40-43

9. Fritze J. Innovative Therapien erfordern zusätzliche finanzielle Ressourcen - Teil II (Antidementiva). Neurotransmitter 2001; 12(2): 30-31

10. Fritze J, Gastpar M. Demenzkranke bitte nicht diskriminieren. Stellungnahme zur Kritik am Wirksamkeitsnachweis von Cholinesterasehemmern. Psychoneuro 2004; 30: 512-513

11. Fritze J, Schmauß M. Off-Label-Use: Der nes S, Kirk C, Waters K, Gardner D, Faulding
Fall Methylphenidat. Psychoneuro 2003; 29 : 302-304

12. Geddes J, Freemantle N, Harrison P, Bebbington P. Atypical antipsychotics in the treatment of schizophrenia: systematic overview and meta-regression analysis. BM] 2000; 321: 1371-1376

13. Gorman JM, Korotzer A, Su G. Efficacy Comparison of Escitalopram and Citalopram in the Treatment of Major Depressive Disorder: Pooled Analysis of PlaceboControlled Trials. CNS Spectrums 2002; 7(suppl.1): 40-44

14. Gunnell D, Middleton N, Whitley E et al. Why are suicide rates rising in young men but falling in the elderly? A time-series analysis of trends in England and Wales 1950-1998. Soc Sci Med 2003; 57: 595-611 15. Hall WD, Mant A, Mitchell PB, Rendle VA, Hickie IB, McManus P. Association between antidepressant prescribing and suicide in Australia, 1991-2000: Trend analysis. Br Med J 2003; 326: 1008-1011

16. Healy D. Lines of evidence on the risks of suizide with selective serotonin reuptake inhibitors. Psychother Psychosom 2003; 72 : 71-79

17. Jureidini JN, Doecke Ch, Mansfiedl $R$, Haby M, Menkes B, Tonkin A. Efficacy and safety of antidepressants for children and adolescents. BM] 2004; 328: 879-883

18. Lepola U, Wade A, Andersen HF. Do equivalent doses of escitalopram and citalopram have similar efficacy? A pooled analysis of two positive placebo-controlled studies in major depressive disorder. Int Clin Psychopharmacol 2004; 19(3):149-155 19. Mann K, Lehert Ph, Morgan MY. The Efficacy of Acamprosate in the Maintenance of Abstinence in Alcohol-Dependent Individuals: Results of a Meta-Analysis. Alcohol Clin Exp Res 2004; 28: 51-63

20. Olfson M, Shaffer D, Marcus SC. Relationship between antidepressant medication treatment and suicide in adolescents. Arch Gen Psychiatry 2003; 60: 978-982

21. Rihmer Z. Can better recognition and treatment of depression reduce suicide rates? A brief review. Eur Psychiat 2001; 16: 406-409

22. Svensson S, Mansfield PR. Escitalopram: superior to citalopram or a chiral chimera? Psychother Psychosom 2004 73(1): 10-16 23. Tariot PN, Farlow MR, Grossberg GT, Graham SM, McDonald S, Gergel I for the Memantine Study Group. Memantine treatment in patients with moderate to severe Alzheimer disease already receiving donepezil. JAMA 2004; 291: 317-324

\section{Korrespondenzadresse:}

Prof. Dr. med. Jürgen Fritze

Asternweg 65

50259 Pulheim 